\title{
Jet substructure in NC DIS at HERA
}

\author{
Elias Ron
}

Universidad Autonoma de Madrid

\begin{abstract}
Jet substructure for jets produced in the neutral current deep inelastic ep scattering regime has been studied for exchanged boson virtualities $Q^{2}>125 \mathrm{GeV}^{2}$ with the ZEUS detector using $368 p b^{-1}$ of integrated luminosity. Jets were identified in the laboratory frame using the $k_{T}$ cluster algorithm in the longitudinally invariant inclusive mode. The substructure of jets has been investigated in terms of the integrated jet shape and was used to highlight differences between quark- and gluon- initiated jets. Monte Carlo models and next-to-leading-order QCD calculations are compared to the data.
\end{abstract}

\section{Introduction}

Jet production in ep collisions provides a fruitful testing ground for perturbative QCD (pQCD). Measurements of differential cross sections for jet production have allowed studies of parton dynamics, tests of the proton parton distribution function (PDFs) as well as determinations of the strong coupling constant, $\alpha_{s}$. This analysis studies the differences in jet substructure for gluon- and quark-initiated jets in the context of ep collisions, providing a more stringent test of the $\mathrm{pQCD}$ predictions.

\section{Theoretical expectations}

At sufficiently high jet transverse energy, the internal substructure of a jet is expected to be calculable in $\mathrm{pQCD}$, since the fragmentation effects become small and thus parton radiation provides the main contribution to jet substructure. PQCD calculations predict that gluoninitiated jets are broader than quark-initiated jets due to the larger color charge of gluons.

\subsection{Integrated Jet shape definition}

In this analysis, the jet substructure of jets is investigated with the integrated jet shape [2], $\psi(r)$, which is defined as the fraction of the total transverse energy of a jet contained within a circle of radius $r$ concentric with the jet center,

$$
\psi(r)=\frac{E_{T}(r)}{E_{T}^{j e t}},
$$

where $E_{T}(r)$ is the transverse energy within the given circle of radius $r$. For a sample consisting of $N_{\text {jets }}$ jets, the average integrated jet shape is:

$$
\langle\psi(r)\rangle=\frac{1}{N_{\text {jets }}} \sum_{\text {jets }} \frac{E_{T}(r)}{E_{T}^{\text {jet }}}
$$

The integrated jet shape is calculated at leading order (LO) in pQCD as the fraction of the jet transverse energy, due to parton emission, that lies between the circles of radii $r$ and $R=1$ :

$$
\langle 1-\psi(r)\rangle=\frac{\int_{r}^{R} d E_{T}\left(E_{T} / E_{T}^{j e t}\right)\left[d \sigma(e p \rightarrow 2 \text { partons }) / d E_{T}\right]}{\sigma_{j e t}\left(E_{T}^{j e t}\right)},
$$


where $\sigma_{j e t}\left(E_{T}^{j e t}\right)$ is the cross section for the inclusive production of jets. In the next-toleading-order (NLO) QCD predictions of the integrated jet shape, the numerator in the above formula is calculated to $O\left(\alpha \alpha_{s}^{2}\right)$ and the denominator to $O\left(\alpha \alpha_{s}\right)$.

\section{$3 \quad$ Data selection and jet search}

The data used in this analysis correspond to an integrated luminosity of $368 \mathrm{pb}^{-1}$.

\subsection{Jet search and samples}

The $k_{T}$ cluster [3] algorithm was used in the longitudinally invariant inclusive mode [4] to reconstuct jets in the laboratory frame. The jet variables were defined according to the Snowmass convention. After jet-energy corrections, events with jets such that $E_{T}^{\text {jet }}>14$ $\mathrm{GeV}$ and $-1<\eta^{j e t}<2.5$ and $-1<\eta^{j e t}<2.5$ were retained. Several jet samples were selected:

- inclusive-jet sample: all jets in such event with $E_{T}^{\text {jet }}>14 \mathrm{GeV}$ and $-1<\eta^{\text {jet }}<2.5$

- one-jet sample: events with exclusively one jet.

- lowest- $E_{T}^{\text {jet }}-D=2.0$ sample: events with exclusively two-jets such that $E_{T}^{j e t_{1,2}}>14$ $G e V,-1<\eta^{j e t}<2.5$, and a distance between them in the $\eta-\phi$ plane $D \leq 2.0$. The jet with lowest $E_{T}^{\text {jet }}$ is considered.

- lowest- $E_{T}^{\text {jet }}-D=2.5$ sample: events with exclusively two-jets such that $E_{T}^{\text {jet }_{1,2}}>14$ $\mathrm{GeV}$ and a distance between them in the $\eta-\phi$ plane $D \leq 2.0$. The jet with lowest $E_{T}^{j e t}$ is considered.

Measurements of the mean integrated jet shape were also made for samples in which the jet transverse energy was restricted to the range $14<E_{T}^{\text {jet }}<17 \mathrm{GeV}$.

In the first two samples of jets, quark-parton-model processes are expected to be dominant, since it is the process responsible for single-jet production. Since the produced jet is a quark-initiated jet, these samples are expected to be enriched with quark-initiated jets. In the other two samples, the contributions from boson-gluon-fusion and QCD-Compton become dominant. By selecting the lowest $E_{T}^{\text {jet }}$, it is expected that the gluon emission by a quark in a QCD-Compton event is enhanced, therefore enriching these samples with gluon-initiated jets.

\section{Next to Leading Order Calculations}

The NLO calculations for the jet substructure have been performed in the laboratory frame, since this is the reference frame where one can have NLO contributions to jet subtructure, that is up to 3 partons in the same jet. For the inclusive-jet and one-jet samples, the program DISENT [5] was used, whereas for the two-jet samples the calculations were done using NLOJET $++[6]$. 

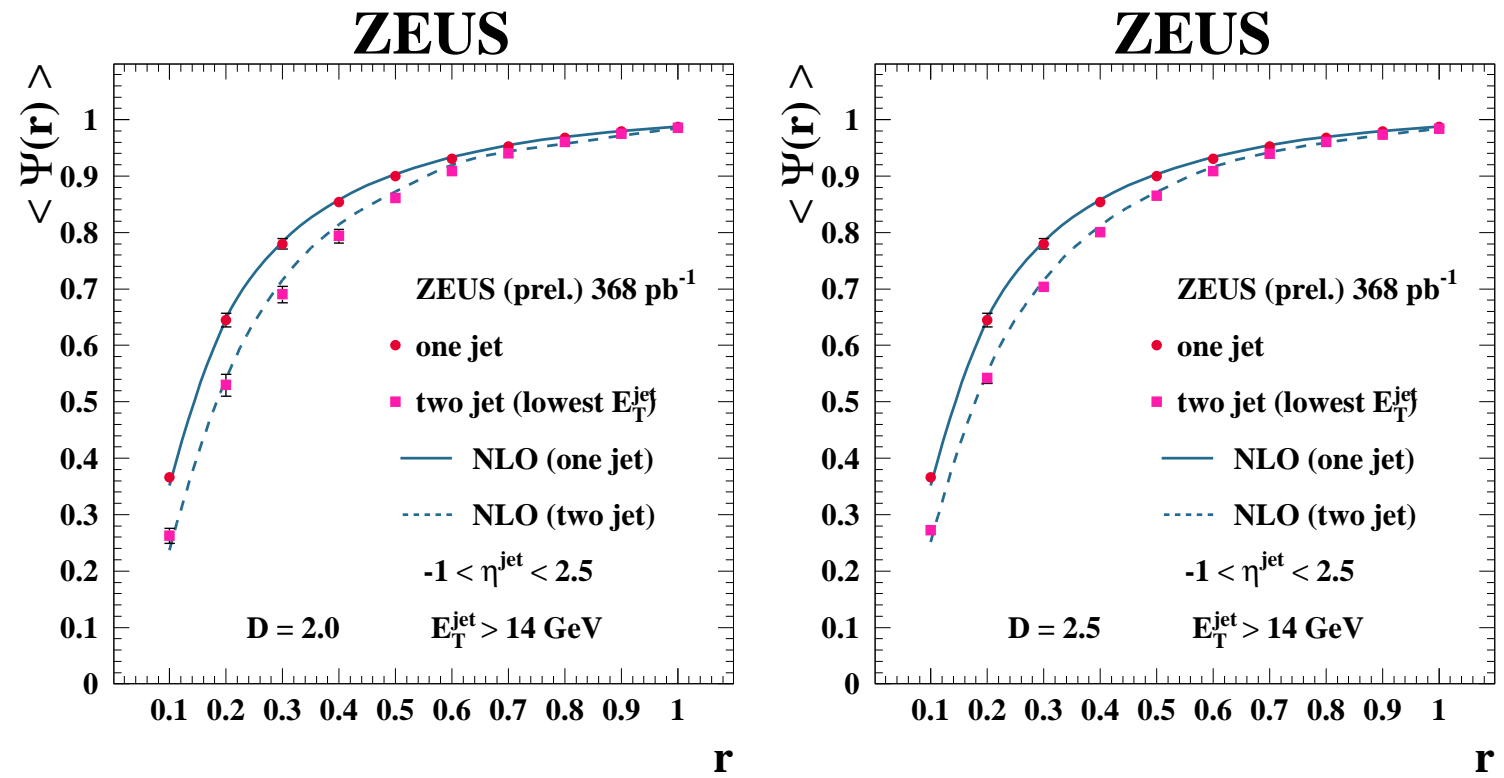

Figure 1: Comparison of the integrated jet shape as a function of $r$ between the onejet sample and the lowest $E_{T}^{j e t}$ jet sample. The data are also compared with the NLO calculations for each sample.

\section{Results}

A comparison of the measured mean integrated jet shape as a function of $r$ between the onejet sample and the lowest $E_{T}^{\text {jet }}$ jet sample is shown if figure 1 , for both values of $D$. As can be seen, jets in the lowest $E_{T}^{\text {jet }}$ jet sample have a broader substructure, which is compatible with the assumption that has a higher content of gluon-initiated jets. The same is observed if the one-jet sample is replaced by the inclusive-jet sample. The data are compared to NLO QCD calculations and good agreement is observed; the calculations are able to reproduce the obverved differences between the one-jet and dijet samples.

However, there is a dependence of the jet substructure with the jet transverse energy; jets get narrower as the transverse energy increases. Thus if the jets in the one-jet and dijet samples were populating different regions in $E_{T}^{j e t}$, the difference in the substructure could arise from this effect. To remove this possible effect, the transverse energy of the jets was restricted between $14 \mathrm{GeV}$ and $17 \mathrm{GeV}$. The corresponding results can be seen in figure 2. The difference between the one-jet and dijet samples still persists and the NLO QCD calculations reproduce this behaviour well. 


\section{ZEUS}

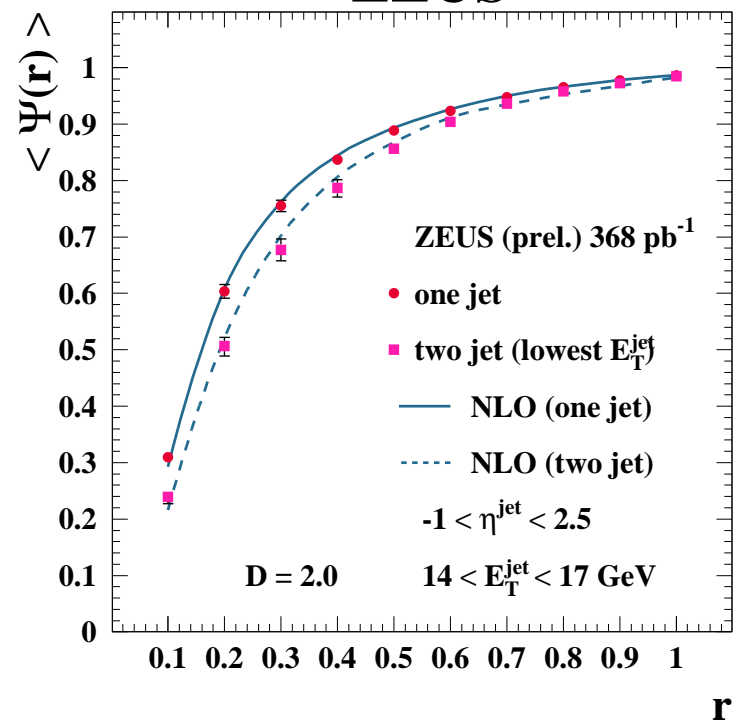

ZEUS

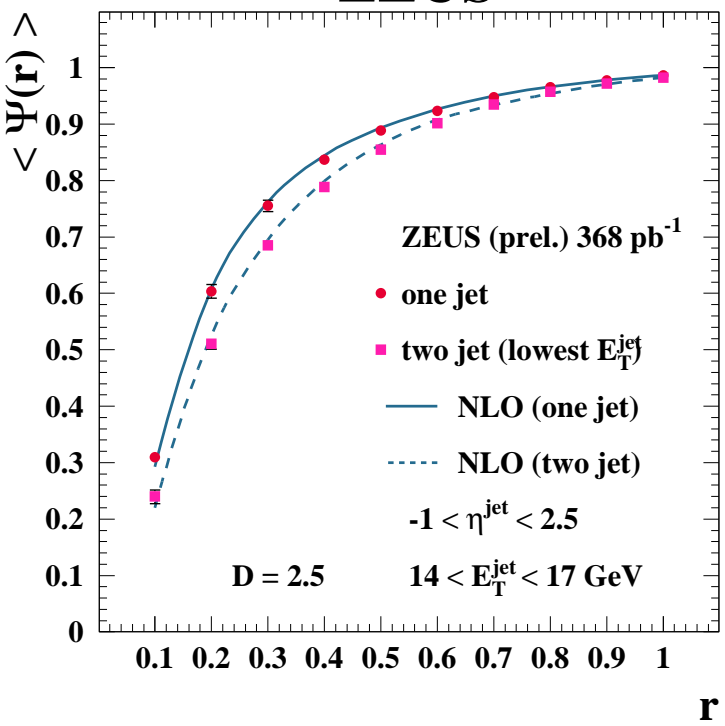

Figure 2: Comparison of the integrated jet shape as a function of $r$ between the one-jet sample and the lowest $E_{T}^{\text {jet }}$ jet sample, for which the transverse energy of the jets has now been restricted between $14 \mathrm{GeV}$ and $17 \mathrm{GeV}$. The data are also compared with the NLO calculations for each sample.

\section{References}

[1] Slides: http://indico. cern. ch/contributionDisplay py? contribId=220\&session $I d=13 \& \operatorname{conf} I d=24657$

[2] S.D. Ellis, Z. Kunszt and D.E. Soper, Phys. Rev. Lett. 69, 3615 (1992)

[3] S. Catani et al., Nucl. Phys. Rev. B 406, 187 (1993)

[4] S.D. Ellis and D.E. Soper, Phys. Rev. D 48, 360 (1997)

[5] S. Catani and M.H. Seymour, Nucl. Phys. B 485, 291 (1997). Erratum in Nucl. Phys. B 510 (1998) 503.

[6] Z.Nagy and Z.Trocsanyi, Phys. Rev. Lett. 87, 082001 (2001). 